\title{
Towards Bridging the Gaps in Holistic Transition Prediction via Numerical Simulations
}

\author{
M. M. Choudhari, ${ }^{1}$ F. Li, ${ }^{2}$ L. Duan, ${ }^{3}$ C.-L. Chang, ${ }^{4}$ M. H. Carpenter, ${ }^{5}$ C. L. Streett, ${ }^{6}$ and M. R. Malik ${ }^{7}$ \\ NASA Langley Research Center, Hampton, VA, 23681
}

The economic and environmental benefits of laminar flow technology via reduced fuel burn of subsonic and supersonic aircraft cannot be realized without minimizing the uncertainty in drag prediction in general and transition prediction in particular. Transition research under NASA's Aeronautical Sciences Project seeks to develop a validated set of variable fidelity prediction tools with known strengths and limitations, so as to enable "sufficiently" accurate transition prediction and practical transition control for future vehicle concepts. This paper provides a summary of selected research activities targeting the current gaps in high-fidelity transition prediction, specifically those related to the receptivity and laminar breakdown phases of crossflow induced transition in a subsonic swept-wing boundary layer. The results of direct numerical simulations are used to obtain an enhanced understanding of the laminar breakdown region as well as to validate reduced order prediction methods.

\section{Nomenclature}

$A \quad=$ amplitude of crossflow instability mode or secondary instability mode, measured in terms of peak chordwise velocity perturbation

$c=$ wing chord length normal to the leading edge

$C \quad=$ coupling coefficient for receptivity

$D \quad=$ diameter of cylindrical roughness element

$f \quad=$ frequency of instability oscillations $(\mathrm{Hz})$

$F \quad=$ forcing function representing external disturbance environment

$\tilde{F} \quad=$ geometry factor from receptivity theory

$G \quad=$ gain factor associated with instability amplification

$i \quad=(-1)^{1 / 2}$

$k=$ grid index along wall-normal direction

$M \quad=$ freestream Mach number

$N=$ N-factor (i.e., logarithmic amplification ratio) of linear crossflow instability or secondary instability

$q \quad=$ arbitrary flow variable

$R \quad=\quad$ transfer function associated with receptivity

$\operatorname{Re}_{c} \quad=$ Reynolds number based on wing chord

$t \quad=$ time

$(u, v, w)=$ Cartesian velocity components aligned with $(x, y, z)$ axes

$x=$ chordwise coordinate in direction perpendicular to leading edge

$y=$ Cartesian coordinate normal to $x$ - $y$ plane

$z=$ spanwise coordinate, i.e., the coordinate parallel to airfoil leading edge

$\alpha=$ chordwise wavenumber

\footnotetext{
${ }^{1}$ Aerospace Technologist, MS 128, Meelan.M.Choudhari@nasa.gov, Associate Fellow, AIAA.

${ }^{2}$ Aerospace Technologist. MS 128, Fei.Li@ nasa.gov.

${ }^{3}$ Aerospace Technologist, MS 128, Lian.Duan@nasa.gov, Senior Member, AIAA.

${ }^{4}$ Aerospace Technologist, MS 128, C.-L.Chang@nasa.gov, Senior Member, AIAA.

${ }^{5}$ Aerospace Technologist, MS 128, M.H.Carpenter@nasa.gov.

${ }^{6}$ Senior Research Scientist, MS 128, C.L.Streett@nasa.gov.

${ }^{7}$ Senior Aerodynamicist, MS 128, Mujeeb.R.Malik@nasa.gov, Fellow, AIAA.
}

1

American Institute of Aeronautics and Astronautics 


$\begin{array}{ll}\beta & =\text { spanwise wavenumber } \\ \Gamma & =\text { wing sweep angle } \\ \lambda & =\text { spanwise wavelength of crossflow instability in millimeters in direction parallel to leading edge } \\ \Lambda & =\text { efficiency function for localized receptivity } \\ \xi & =\text { dummy integration variable in chordwise direction } \\ \pi & =\text { normalized mode shape of instability wave } \\ \theta & =\text { phase function } \\ \phi & =\text { normalized mode shape of instability wave } \\ \omega & =\text { radian frequency of instability wave }\end{array}$

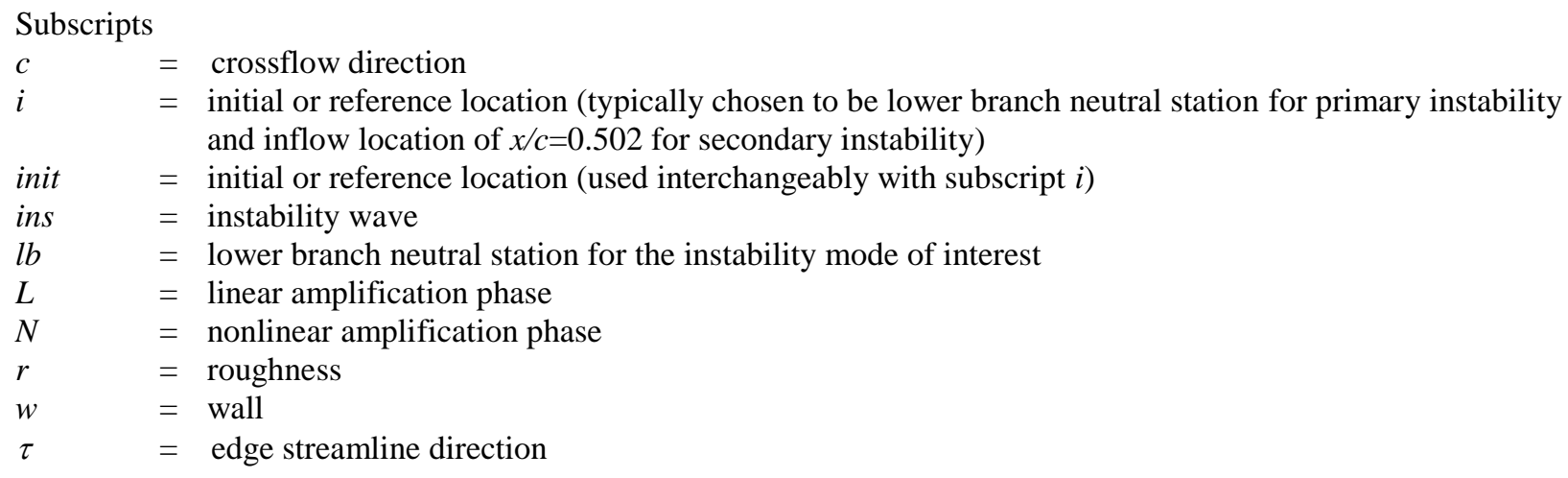

\section{Introduction}

UBSTANTIAL reductions in the fuel burn of future aircraft have been targeted both in the U.S. ${ }^{1}$ and in Europe ${ }^{2}$ S in order to lower the cost of air travel and alleviate the impact of aviation on the environment. A significant fraction of the targeted reductions in aircraft fuel burn will come from the reductions in vehicle drag. For today's commercial transport aircraft, up to one half of the total drag corresponds to skin friction drag. Because laminar skin friction is much lower than the turbulent skin friction, flow control via delayed boundary-layer transition over major aerodynamic surfaces holds the potential to provide significant reductions in the overall drag. For example., savings in the range of 8.7-13.5 percent have been estimated to result via the implementation of laminar flow technology on future aircraft with a projected entry-in-service date of $2025 .^{3}$

NASA's Subsonic Fixed Wing (SFW) Project investigated several options to help develop robust technology concepts for laminar flow control and to accelerate their insertion into the operational fleet. ${ }^{1}$ One of those concepts involved the delay of crossflow-induced transition over a swept aerodynamic surface by using control input in the form of appropriately positioned Discrete Roughness Elements (DREs) near the leading edge. ${ }^{4}$ Most of the previous experimental and computational studies of the DRE concept had been carried out for low-subsonic Mach number configurations with modest wing-chord Reynolds numbers of up to approximately eight million, ${ }^{5,6}$ and with pressure distributions that may not be optimal for wing designs for subsonic transport aircraft flying at Mach numbers between 0.75 to 0.90 . Consequently, the studies at both Texas A \& M University ${ }^{7}$ (TAMU) and NASA ${ }^{8}$ under the SFW project focused on assessing the potential capability of the DRE concept to control swept wing transition at transonic Mach numbers and substantially higher chord Reynolds numbers than previous applications. This work

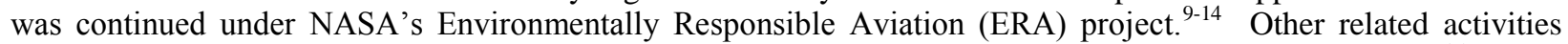
under the SFW project focused on in-flight characterization of crossflow receptivity to surface roughness, ${ }^{15}$ design tools $^{16}$ and high Reynolds number testing for natural laminar flow (NLF) wings. ${ }^{17}$ These activities were subsequently transitioned to the ERA project. The recently initiated Aeronautical Sciences (AS) project of NASA is aimed at developing computer-based tools and models as well as scientific knowledge that will lead to significant advances in our ability to understand and predict flight performance for a wide variety of air vehicles. Transition research under the AS project seeks to develop a validated set of variable fidelity prediction tools with known strengths and limitations, so as to enable sufficiently accurate transition prediction and practical transition control for future vehicle concepts. ${ }^{18}$ The objective of this paper is to present the initial results obtained under this effort. 

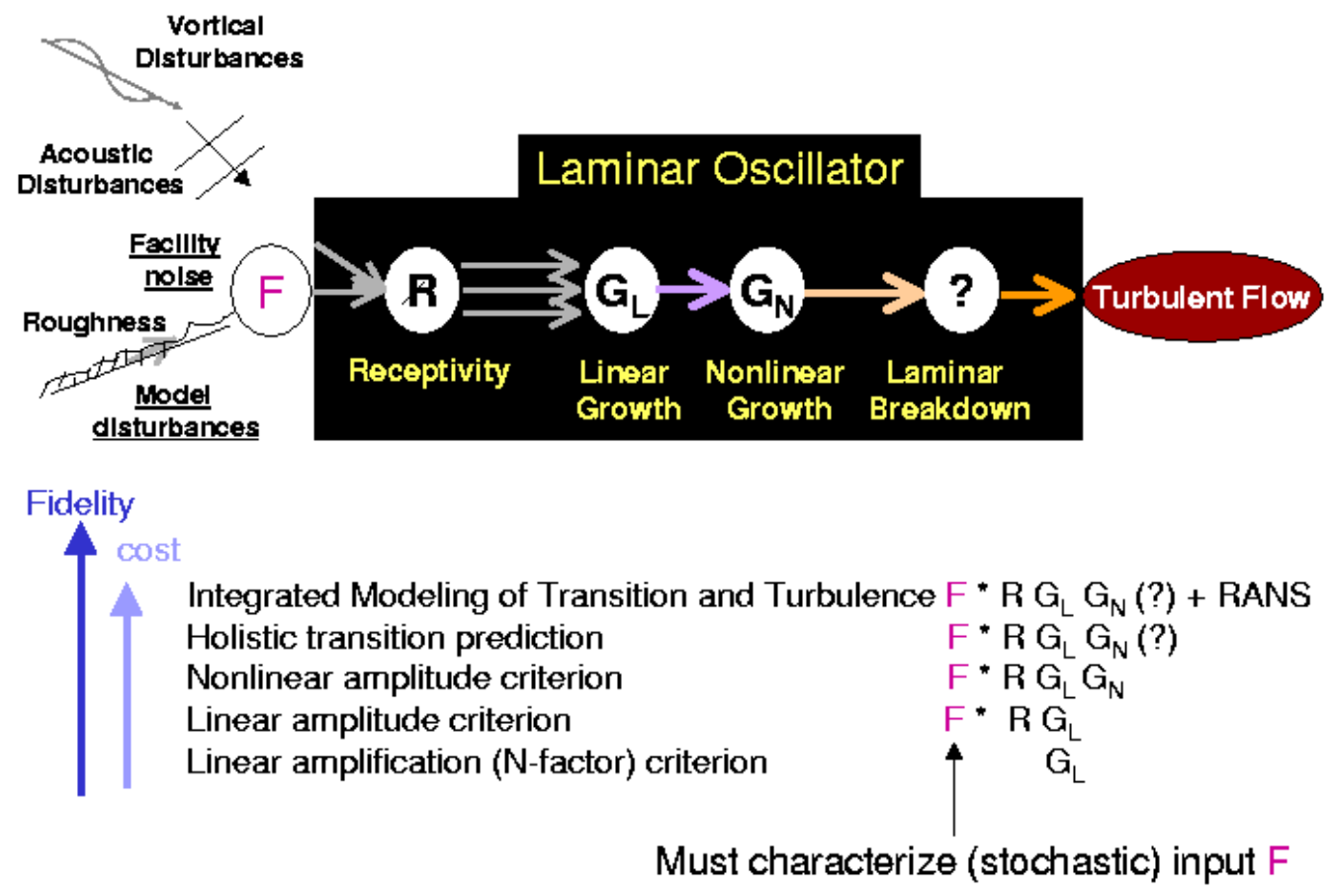

Figure 1. Schematic of hierarchical levels of transition prediction from a systems viewpoint. ${ }^{19}$ The holistic prediction approach, based on transition as a forced response of a nonlinear system to stochastic forcing, appears near the top of this hierarchy. The stagewise transfer functions (i.e., gain factors) associated with receptivity, linear amplification, and nonlinear growth are denoted by $R, G_{L}$, and $G_{N}$, respectively. The parenthesized question mark denotes the lack of reliable methods to bridge the gap between physics based modeling preceding the laminar breakdown phase and some form of statistical turbulence modeling farther downstream.

Given the sensitivity of swept-wing transition to wind tunnel disturbances and the cost of flight experiments, computational tools will need to play an important role in the assessment of DREs for subsonic transport vehicles as a means of risk reduction as well as to help optimize the DRE design. The DRE concept seeks to delay transition via controlled seeding of subdominant crossflow modes that cannot lead to transition on their own, but can keep the naturally dominant instability modes at bay via nonlinear modification of the basic state and/or mode competition. This intrinsically nonlinear control mechanism calls for a higher-fidelity prediction approach involving all of the relevant stages of the transition process, namely, receptivity, linear, and nonlinear growth of primary crossflow instabilities and secondary instability. ${ }^{19,20}$ (See Fig. 1 for a schematic of physics based transition prediction based on increasing model fidelity.) The individual ingredients of this holistic approach have been available for some time and partial integration of those ingredients has been demonstrated in prior works. ${ }^{19-26}$ In particular, computations of this type for the low Mach number configuration used during the flight experiment by TAMU at chord Reynolds numbers between seven and eoght million have been reported previously.,26,27 Results based on a combination of nonlinear parabolized stability equations (NPSE) and linear secondary instability theory (LSIT) showed that the most unstable stationary crossflow mode is weakened in the presence of artificially introduced roughness elements, with an accompanying reduction in the growth potential of the high-frequency secondary instability modes that are likely to cause the onset of laminar-turbulent transition. ${ }^{26}$ However, the same results also indicated that the control mode amplitudes required to stabilize the dominant modes can become excessively large, especially when the maximum $\mathrm{N}$-factors of the primary crossflow instability are rather large, so that the possibility of premature transition due to overcontrol cannot be ruled out. Similar findings had previously been obtained for a supersonic swept wing configuration at Mach 2.4 and chord Reynolds number of 16 million. ${ }^{23,24}$

The aforementioned SFW and ERA efforts targeting passive laminar flow over a subsonic transport involved a series of conceptual design studies of a laminar flow control (LFC) flight test. The initial studies under SFW ${ }^{7,8}$ 
focused on conditions relevant to a subsonic transport, namely, a flight Mach number of 0.75 , a chord Reynolds number, $\mathrm{Re}_{\mathrm{c}}$, between 15 and 20 million, and a leading-edge sweep angle, $\Gamma$, of $30^{\circ}$. The resulting wing design was predicted to have a significant potential for natural laminar flow over both suction and pressure surfaces at the design angle of attack of zero degrees. At off-design conditions, however, earlier transition was deemed likely as a result of stationary crossflow modes. This finding opened up the possibility of DRE-based transition control on the same airfoil, either to increase the lift coefficient or to extend the angle-of-attack range for laminar flow. ${ }^{7}$ The companion study by Li et al. ${ }^{8}$ sought to assess the feasibility of achieving DRE-based transition control by using NASA's prediction tools for nonlinear development and secondary instability stages of the transition process and to help mature those prediction tools and the methodology to apply them in a practical context. The latter results are extended in this paper (as well as in a companion paper $^{27}$ ) to address the receptivity phase (i.e., roughness-induced excitation of stationary crossflow modes), non-parallel and nonlinear effects on the growth of high-frequency secondary instabilities of stationary crossflow modes, and the subsequent breakdown process.

In the interest of making this paper self-contained, a brief description of the flow configuration as well as the computational methodology is given in Section II below. Results pertaining to the excitation of stationary crossflow vortices due to deterministic but spatially distributed surface roughness (waviness) are outlined in Section III. Section IV presents the findings from direct numerical simulations aimed at validating the previous predictions based on both LSIT and parabolized stability equations (PSE) which pertain to the amplification of secondary instability of stationary crossflow vortices. Section V outlines a specific scenario for the nonlinear breakdown of secondary instability modes resulting in a turbulent, swept-wing boundary layer. To gain a further understanding of the role of traveling crossflow modes in the transition of a swept wing boundary layer, the secondary instability characteristics of finite amplitude traveling crossflow modes are described in Section VI. Concluding remarks are presented in Section VII.

\section{Flow Configuration and Analysis Codes}

The design process for the laminar flow airfoil TAMU-003T-75(v.90) was described by Belisle et al. ${ }^{7}$ The 9.3 percent thick, 30-degree swept airfoil is designed to achieve natural laminar flow over 60 and 50 percent of the suction and pressure surfaces, respectively, at the design condition of zero degree angle of attack, Mach number of $\mathrm{M}=0.75$, and chord Reynolds number of approximately 17 million based on freestream speed, a streamwise chord

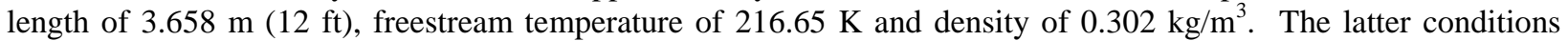
correspond to a flight altitude of 12,192 m (40,000 ft). Design constraints for the wing included a lift coefficient that is typical of subsonic transports and a wing thickness distribution that is suitable for a mid-size business jet. At the design angle of attack, both Tollmien-Schlichting and crossflow instabilities are sufficiently weak that natural laminar flow should be achievable over a significant portion of the airfoil surface $(x / c>0.6$ on the suction surface and $x / c>0.5$ on the pressure side) without any external means of boundary layer control. In this paper, we focus on the off design condition corresponding to an angle of incidence equal to -1 degree, i.e., the same condition as that used by Li et al. ${ }^{8}$ in their PSE and LSIT analysis. At this off-design condition, a stronger crossflow instability exists on the suction side, ${ }^{7,8}$ which provides the basis for potentially implementing DRE-based laminar flow technology at the higher Reynolds numbers of interest.

For the work described in this paper, the mean boundary-layer flow over the suction surface of the airfoil was obtained from the boundary layer solution obtained by Li et al. ${ }^{8}$ It was computed using a boundary layer solver BLSTA ${ }^{28}$ designed specifically for generating accurate boundary layer solutions that are required for boundary layer stability analysis of swept and tapered wings, in conjunction with the inviscid surface-pressure distribution derived from an Euler solution under free flight conditions. ${ }^{7}$ The number of wall-normal points used for boundary layer computations was well in excess of that used by $\mathrm{Wie}^{28}$ for validating the BLSTA code. Sensitivity to streamwise resolution was also assessed by $\mathrm{Li}$ et al. ${ }^{8}$ by using $115,229,457$ and 913 points over the chord length of interest (with proportionally greater number of points near the leading edge), and the solution was converged with 115 streamwise points. Similar to the preceding work, ${ }^{8}$ the computations presented in this paper are based on 229 points over the chord length. The boundary layer solution computed in this manner is suitably interpolated using cubic splines when necessary.

The growth of high-frequency secondary instability modes supported by the finite amplitude stationary crossflow vortex was analyzed by $\mathrm{Li}$ et al. ${ }^{8}$ with a fully spatial methodology described in earlier papers ${ }^{23,24}$ and their predictions for the case of Ainit $=1 \times 10^{-5}$ are compared with the DNS results in Section IV. The main difference between the primary and secondary stability analyses is that the basic state for the secondary modes (i.e., the mean boundary layer flow modified by the primary crossflow mode) varies in both surface normal and spanwise directions; and hence, the instability characteristics of the secondary modes must be analyzed using a planar, partial 
differential equation based eigenvalue problem, rather than an ordinary differential equation based eigenvalue problem of the classical analysis. The selection of grid and other aspects of the numerical solution was based on extensive experience with a similar class of flows and spot checks were made to ensure that the impact of reasonable variations with respect to those choices was negligible. Typically, 121 points in the wall-normal direction and 33 points in the spanwise direction were used for the 2D eigenvalue analysis. A similar methodology and spatial resolution are used in Section VI to analyze the secondary instability of traveling crossflow modes.

Linear and nonlinear development of the primary instability (i.e., either stationary or traveling crossflow modes) was computed using linear/nonlinear parabolized stability equations (LPSE and NPSE, respectively) as implemented in the Langley Stability and Transition Analysis Codes (LASTRAC). ${ }^{29}$ For nonlinear PSE marching, 281 points are used in the wall-normal direction (which is more than sufficient), and the streamwise marching step-size was appropriately adjusted for each computation, if necessary, to capture the corresponding modal evolution. We denote the ranges of Fourier modes in the spanwise direction and in time by $(-m, m)$ and $(-n, n)$, respectively. A total of 48 spanwise Fourier harmonics $(m=48)$ is used to compute the nonlinear development of a primary crossflow mode of specified spanwise wavelength, $\lambda$, and the truncation error is estimated a posteriori to be at least 9 orders of magnitude smaller than the most energetic fundamental mode.

Receptivity calculations are performed using the adjoint PSE module implemented within the LASTRAC code. ${ }^{30}$ The spatial resolution used for the adjoint PSE computations is similar to that used for the regular LPSE computations.

To study the breakdown of finite-amplitude crossflow vortices due to high-frequency secondary instability, a direct numerical simulation (DNS) is performed by solving the Navier-Stokes equations in generalized curvilinear coordinates. The working fluid is assumed to be an ideal gas with linear Newtonian strain-stress relation. The Fourier law is used for the heat flux. The computational domain is approximately aligned with the axis of the primary crossflow mode and extends over more than 100 wavelengths of the secondary instability wave at a frequency of $42.5 \mathrm{kHz}$. The size of the computational grid corresponds to $5,000 \times 242 \times 307$ points in the chordwise, spanwise, and wall-normal directions, respectively. In terms of wall units of the fully turbulent flow near the outflow of the domain, the grid resolution in the streamwise $(x)$ and spanwise directions $(y)$ corresponds to $\Delta x^{+} \approx$ 10 and $\Delta y^{+} \approx 4.5$, respectively. The first grid point in the wall normal direction is located at $\Delta z^{+} \approx 0.6$.

A $7^{\text {th }}$-order weighted essentially non-oscillatory (WENO) $s c h e m e^{32,33}$ is used to compute the convective flux terms. This particular WENO scheme combines a high order of accuracy with relatively low dissipation, making it suitable for simulations of compressible transitional flows. The resolution properties of this scheme are documented in many references, e.g. Martin et al. ${ }^{34}$ For the viscous flux terms, a $4^{\text {th }}$-order central difference scheme is used and the $3^{\text {rd }}$-order low storage Runge-Kutta scheme by Williamson ${ }^{35}$ is employed for time integration. Unsteady nonreflecting boundary conditions based on Thompson ${ }^{36}$ are imposed at the freestream boundary. At the outflow boundary, a buffer domain technique is used before the primitive variables are extrapolated at the outflow plane. The DNS code has been previously shown to be suitable for computing fully turbulent flows. ${ }^{34,37}$ In additional unpublished work, further validation of this code was performed in the context of the propagation of linear instability waves in a 2D, high-speed boundary layer. Convergence studies showed that the $7^{\text {th }}$-order WENO performs well at a resolution of 10 points per wavelength or higher, depending on the streamwise extent of the computational domain.

\section{Receptivity Characteristics}

Even though the $\mathrm{N}$-factors for stationary crossflow vortices are lower than those of traveling crossflow modes, the inevitable imperfections in surface finish lead to preferential excitation of stationary modes. ${ }^{38,39}$ As a result of their higher initial amplitudes, these stationary modes tend to dominate the crossflow transition process in a benign disturbance environment analogous to that in flight. For cases where the roughness amplitude is sufficiently small and the wavenumber spectrum of the surface height perturbations overlaps with the length scales of the crossflow vortex instabilities, the resulting receptivity can easily be predicted by extending the Goldstein-Ruban theory ${ }^{40,41}$ for localized receptivity. Thus, the flow perturbations associated with an arbitrary crossflow mode of spanwise wavenumber, $\beta$, can be expressed in the form ${ }^{42}$ :

$$
q(x, y, z ; \omega, \beta)=C(x, \omega, \beta) \phi(x, y) \exp \left(i \int_{x_{0}}^{x} \alpha d \xi+\beta z-\omega t\right)
$$

where the cumulative coupling coefficient for distributed receptivity, $C$, corresponds to the effective instability amplitude at a selected reference location $x=x_{0}$. The quantity $\phi(x, y)$ denotes a normalized mode shape associated 
with the perturbations produced by the instability mode. As a result of the assumed linearity of the receptivity process, the coupling coefficient can be expressed as:

$$
\begin{aligned}
& C=\frac{1}{\sqrt{2}} \stackrel{x}{x}_{0}(, \quad) \tilde{F}(,) \exp \left(i_{\text {ins }}(, \quad)\right) d \\
& \text { ins }(x, \quad)=\stackrel{x}{x}_{0} \text { ins }(, \quad) d
\end{aligned}
$$

where $\Lambda(\xi, \beta)$ characterizes the efficiency of the local receptivity to surface roughness, i.e., the local Green's function with the response measured at the forcing location, $\xi$. The geometry factor $\tilde{F}$ represents the Fourier coefficient of the surface roughness distribution corresponding to the spanwise wavenumber $\beta$. The decoupling between geometry and efficiency functions was first pointed out by Goldstein in his pioneering work for localized excitation of Tollmien-Schlichting waves. ${ }^{40}$ Computing the efficiency function for a given mean flow and wave number thus provides a basis for further predicting the disturbance amplitude associated with a surface nonuniformity of finite spatial extent.

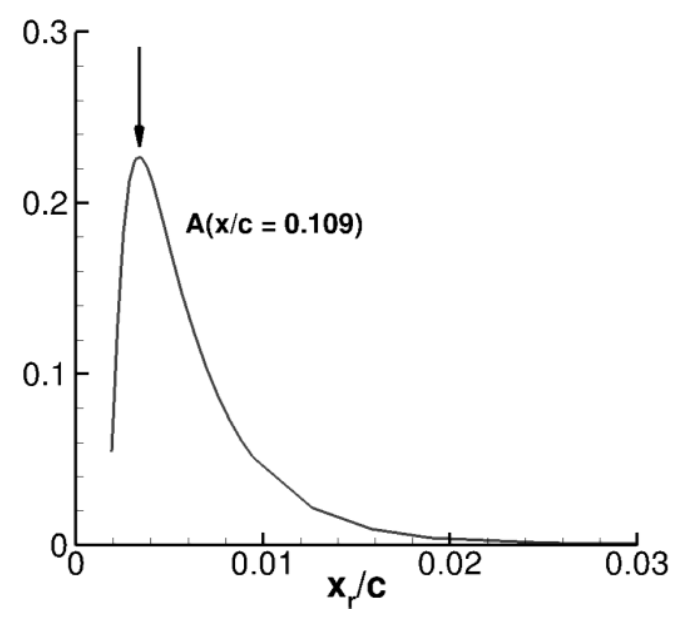

Fig. 2. Localized receptivity due to a single array of roughness dots mounted near the leading edge of the TAMU-003T-75(v.90) airfoil: Effect of array location, $x_{r}$, on the amplitude of stationary crossflow vortex. $\left(\lambda=2.67 \mathrm{~mm} ; D_{r}=1.33 \mathrm{~mm}\right.$; and the height of roughness dots is assumed to be $1.0 \mu \mathrm{m}$.)

For the TAMU-003T-75(v.90) airfoil configuration, ${ }^{7}$ the stationary crossflow vortices that achieve the highest linear amplification at the off design condition of interest correspond to a spanwise wavelength of approximately eight $\mathrm{mm} .{ }^{7,8}$ The $\lambda=2.67 \mathrm{~mm}$ mode has a linear $\mathrm{N}$ factor of approximately six and was shown to be suitable as a control input based on DREs. ${ }^{8}$ The excitation of the control mode using a spanwise periodic array of cylindrical roughness elements (i.e., DREs) of diameter $D_{r}=1.33 \mathrm{~mm}$ is considered first. Fig. 2 shows the magnitude of the coupling coefficient plotted as a function of the chordwise location $x_{r}$ of the array of roughness elements. The peak effective receptivity occurs when the DREs are placed near $\mathrm{x} / \mathrm{c}=0.0033$, i.e., near the lower branch location for $\lambda=$ $2.67 \mathrm{~mm}$.

The stationary crossflow mode with $\lambda=8 \mathrm{~mm}$ is excited as part of the flow response to naturally occurring surface roughness, which in principle may include both deterministic and random components. As described by Choudhari, ${ }^{43}$ the receptivity to random distributed roughness can be evaluated using the solution for receptivity to a wavy wall as a building block component. Computational predictions for receptivity to naturally occurring, stochastic roughness distribution over a flight article were presented by Carpenter et al. ${ }^{44}$ Receptivity to wavy walls was also considered in the latter paper, but those results were restricted to the canonical problem of a swept Hiemenz boundary layer. As previously shown by Collis and Lele, ${ }^{45}$ the receptivity to localized roughness elements is strongly influenced by the curvature of the airfoil surface. Thus, it is useful to examine the distributed receptivity due to wavy undulations of small amplitude in the airfoil surface. Figure 3 shows the coupling coefficient for a stationary crossflow vortex with $\lambda=8 \mathrm{~mm}$, plotted as a function of the chordwise wavenumber, $\alpha_{w}$, of the wavy surface height distribution. Again, similar to the canonical case of a swept Hiemenz boundary layer, the peak 
receptivity levels are confined to a narrow band of surface wavenumbers. The wavenumber corresponding to the maximum coupling coefficient is close to the neutral wavenumber of the stationary crossflow mode with $\lambda=8 \mathrm{~mm}$, but is slightly detuned from it. Similar to the swept Hiemenz flow, the wavenumber bandwidth of the flow response is substantially larger than that of acoustic excitation of T-S waves through scattering by the surface waviness. ${ }^{42}$

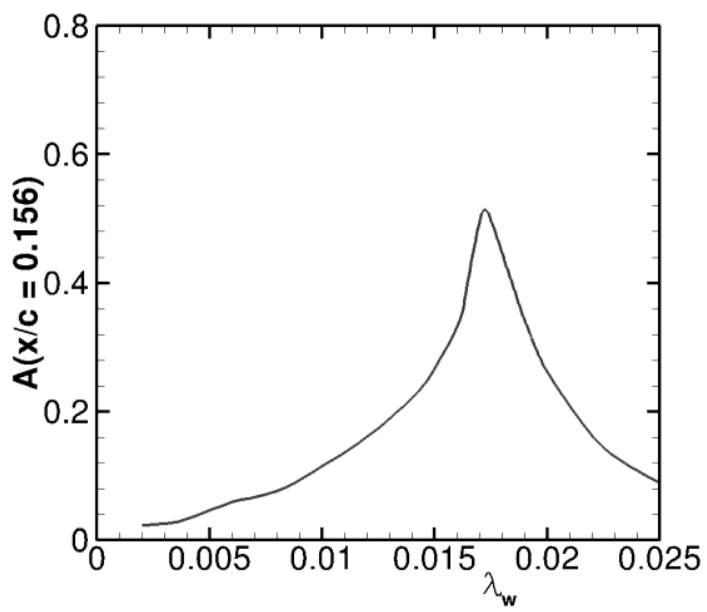

Fig. 3. Receptivity of boundary layer flow over the TAMU-003T-75(v.90) swept airfoil configuration to wavy wall roughness height distribution with peak height of $1.0 \mu \mathrm{m}$ : Effect of surface wavelength on the amplitude of stationary crossflow vortex with $\lambda=8.0$ mm.

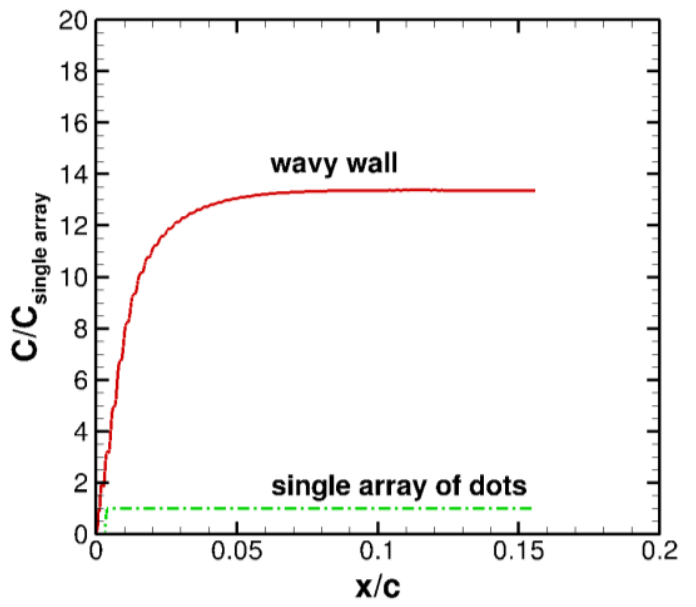

Fig. 4. Distributed receptivity due to wavy wall compared with receptivity due to a single array of roughness dots at the lower branch neutral station $\left(D_{r}=1.33 \mathrm{~mm}\right.$ and the height of the dots is assumed to be the same as the amplitude of the wavy wall).

To ascertain the relative receptivity levels for surface waviness relative to those for a single isolated region of surface imperfection, the cumulative coupling coefficient for $\alpha_{w}=\alpha_{w}, \max =363.2 \mathrm{~m}^{-1}$ (which corresponds to the peak of the response curve in Fig. 3) is plotted as a function of chordwise location in Fig. 4. For reference, the coupling coefficient for a local imperfection corresponding to a single array of cylindrical roughness elements centered on the lower branch station is also shown in this figure. The diameter of the dots is assumed to equal one half of the spanwise wavelength. For $\alpha_{w}=\alpha_{w, \max }$, the stationary crossflow perturbations due to portions of the wavy surface in the vicinity of the lower branch tend to reinforce each other with a nearly zero cancellation. Thus, the cumulative response is approximately one order of magnitude larger than that of a single array of roughness elements centered at the neutral station. The resulting values of coupling coefficient indicated that the initial crossflow amplitudes considered in the NPSE calculations of Li et al. can easily be excited by a wavy wall height distribution at the peak wavenumber even if the root-mean-square height of this distribution is just a few microns.

\section{Non-Parallel and Nonlinear Effects on Secondary Instability Amplification}

Li et al. ${ }^{8}$ performed NPSE computations to predict both isolated and combined nonlinear evolution of the target $(\lambda=8 \mathrm{~mm})$ and control $(\lambda=2.667 \mathrm{~mm})$ modes of the primary, i.e., stationary crossflow instability for varying initial amplitudes. To obtain a greater insight into the expected transition locations, secondary instability analysis was performed using the NPSE results as the modified basic state. Both LSIT and PSE analyses were used to characterize the $\mathrm{N}$-factor evolution of secondary instability modes over a broad range of frequencies. In most cases, the modified basic state supported multiple families of secondary instabilities that derived their amplification from turbulence production mechanisms associated with wall-normal and/or spanwise shear of the basic state. In the notation of Ref. 8, these modes are referred to as the Y- and Z-modes, respectively; and we will continue to employ that nomenclature in this paper. For $\lambda=8 \mathrm{~mm}$, the secondary instability modes associated with wall-normal shear (i.e., Y-mode in the notation of Ref. 8) had the higher amplification rates and, therefore, might be expected to dominate the laminar breakdown process over the swept airfoil configuration examined in this paper. This breakdown process is examined in a companion paper by Duan et al. ${ }^{27}$

The receptivity mechanisms associated with the secondary instability modes are not well understood at this point; and therefore, it is conceivable that, under certain circumstances, the secondary instability modes with lower amplification factors might get excited more strongly than the modes with dominant linear growth and hence cause 
the onset of transition. Indeed, there is some evidence based on prior experiments ${ }^{46}$ and computations ${ }^{47}$ that the secondary instability modes associated with spanwise shear (i.e., Z-mode) may become relevant to the transition process even when their amplification factors are lower than the wall-normal-shear modes. Furthermore, for the airfoil configuration and disturbance parameters examined by Malik et al., ${ }^{14}$ the Z-mode of secondary instability had higher peak amplification factors than the Y-mode. Thus, it is also important to consider the linear and nonlinear evolution of the Z-modes.

According to the LSIT analysis of Li et al., ${ }^{8}$ a spanwise shear mode of frequency $42.5 \mathrm{kHz}$ achieved peak linear $\mathrm{N}$-fators approaching seven and, therefore, could cause transition if it had a sufficiently large initial amplitude. To validate the LSIT and PSE predictions, DNS computations were performed for two different initial amplitudes that differed by a factor of more than 150 . The smaller initial amplitude $\left(A_{\text {init }}=3.4 \times 10^{-5}\right)$ was chosen to mimic the linear growth of the secondary instability mode, whereas the higher initial amplitude $\left(A_{\text {init }}=0.0055\right)$ was chosen to capture nonlinear modification of the initial growth, before a breakdown to turbulence. Fig. 5 displays the amplitude evolution (based on the peak chordwise velocity fluctuation over the cross-section of the primary crossflow vortex) from the two DNS computations and their comparison with the LSIT and PSE predictions of Li et al. ${ }^{7}$ The DNS $^{-1}$ result for the smaller initial amplitude agrees very well with the PSE prediction, confirming that the differences between the LSIT and PSE results are caused by neglecting the weakly nonparallel effects in the LSIT predictions. Furthermore, the DNS results for the higher initial amplitude confirm the initially stabilizing effect of nonlinearity on the fundamental mode amplitude, which is analogous to the finding of Li et al. ${ }^{8}$ The approach to quasi-saturated behavior in the fundamental mode evolution is followed by a rapid increase in the modal amplitude and then a stochastic behavior that is suggestive of the onset of transition. The details of this transition zone are further explored in Section V below.

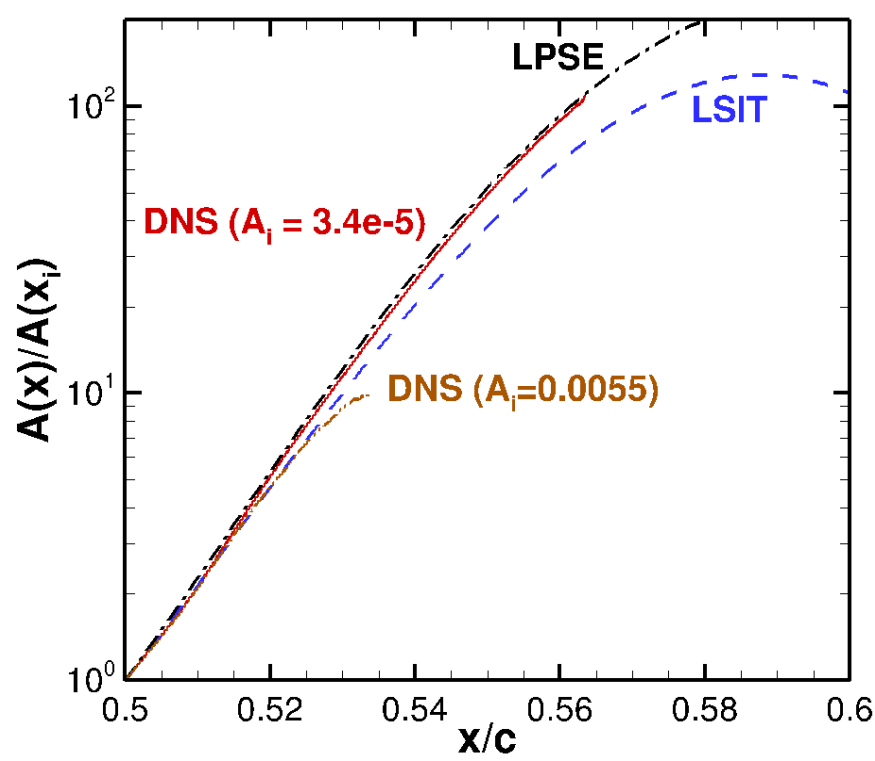

Fig. 5. Amplification curve for Z-mode of secondary instability: Comparison of DNS solutions with LSIT and PSE predictions

\section{Laminar Breakdown due to Secondary Instability of Stationary Crossflow Vortices}

Li et al. ${ }^{8}$ performed NPSE computations to track the joint nonlinear evolution of a stationary crossflow mode with $\lambda=8 \mathrm{~mm}$ and $A_{\text {init }}\left(x_{l b}\right)=1 \times 10^{-5}$ and its secondary instability modes of varying initial amplitudes upto the location where the mean skin friction began to rise rapidly. However, the details of the transition zone could not be inferred due to the limitations of the PSE approach. In this section, the results of the DNS computation involving the same primary mode and a spanwise-shear type (i.e., Z-mode) secondary instability with $f=42.5 \mathrm{kHz}$ and $A_{\text {init }}$ $(x / c=0.502)=0.0055$ are used to explore the boundary layer evolution through the laminar breakdown region. Due to the large computational grid and correspondingly long time interval required to average over a large number of eddies, fully converged turbulence statistics have not been acquired thus far. However, a comparison of instantaneous, spanwise-averaged statistics at different instants of time indicates that the primary conclusions presented herein may be expected to remain unchanged with further accumulation of time history data. Unless 
otherwise specified, all of the flow statistics presented herein have been averaged over 20 periods of the fundamental secondary mode.

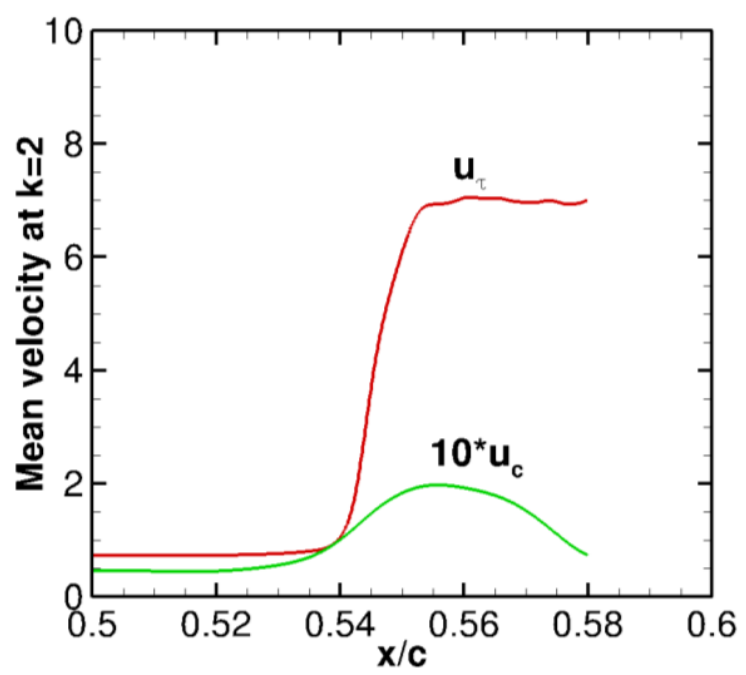

Fig. 6. Dimensional mean wall velocity at first point off the wall (which is located at a fixed wall-normal distance over the entire length of the computational domain). Due to averaging of flow statistics over a limited time interval, additional moving averaging in $x$ has been performed to smooth short scale oscillations in the evolution of flow statistics. Subscripts $\tau$ and $c$ indicate velocity components aligned with the edge streamline and crossflow direction, respectively.

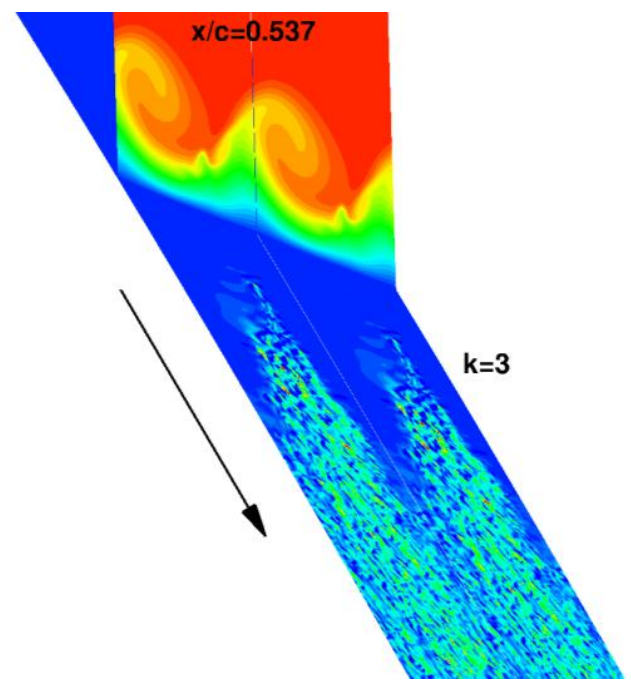

Fig. 7. Instantaneous chordwise velocity contours over both the horizontal coordinate plane $k=3$ (which lies very close to the wall) and the vertical plane $x / c=0.537$ (which lies just upstream of the onset of transition).

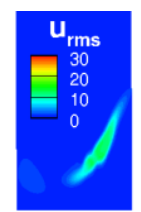

$x / c=0.534$
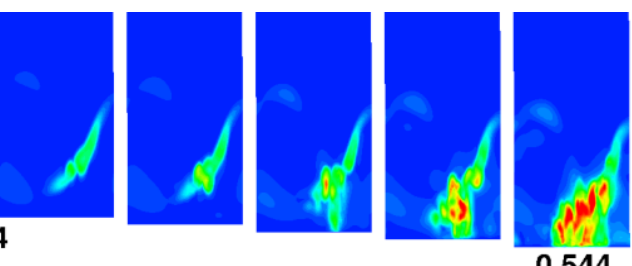

Fig. 8. RMS fluctuations in chordwise velocity component across the cross-section of the stationary vortex at selected stations $(x / c \leq 0.544)$. The RMS is taken over 20 periods of the secondary instability wave.

The chordwise evolution of near-wall mean velocities at a fixed distance above the surface (Fig. 6) indicates a rapid rise in the near-wall mean velocity close to $x / c=0.54$, indicating the onset of transition location. The skin friction reaches a peak near $x / c=0.55$ and then decreases weakly over the remaining length of the computational domain. No prominent overshoot in skin friction is observed in this particular case. Whether the absence of an 
overshoot in the skin friction is caused by the spanwise averaging to obtain the result in Fig. 6 or whether it is a genuine feature of the present scenario for stationary crossflow breakdown remains to be seen. Flow statistics over a longer time interval will be used in the future to clarify whether the overshoot is also absent from the chordwise evolution of mean wall shear within individual spanwise planes. Fig. 6 indicates that the wall shear associated with the crossflow profile also increases after the onset of transition. However, the magnitude of this increase is weaker in comparison with the wall shear along the direction of the inviscid streamline. Moreover, the eventual decrease in the wall shear associated with the crossflow profile implies that the wall shear direction approaches the direction of the streamline at the edge of the boundary layer as the flow goes through transition. The latter finding, originally noted by Huai et al ${ }^{48}$ appears to be associated with the decrease in both crossflow magnitude and the crossflow shear, which corresponds to a shift towards increased two-dimensionality in the mean turbulent flow.

Fig. 7 displays the spanwise variation in instantaneous contours of chordwise velocity distribution over the $\mathrm{k}=3$ surface, which is located at a fixed distance from the airfoil surface. The steep gradients in chordwise velocity contours resemble the sawtooth transition front that is commonly seen in naphthalene-based surface flow visualizations of crossflow transition dominated by stationary vortices. The origin of the turbulent wedge lies within a spanwise region that corresponds to relatively smaller boundary layer thickness, and the completion of the transition process (i.e., the location of peak mean wall shear in Fig. 6) approximately coincides with the merging of adjacent pairs of turbulent wedges. The half-angle of the turbulent wedge inferred from the spanwise wavelength of the stationary crossflow vortex and the cordwise extent of breakdown region corresponds to approximately six to seven degrees. A similar sawtooth pattern has also been observed by Duan et al. ${ }^{27}$ in their DNS of transition caused by the Y-mode of secondary instability (namely, the mode that is primarily associated with the wall-normal shear of the basic state). The similarity between the sawtooth patterns observed in both simulations indicates that the mechanism behind the breakdown of stationary crossflow vortices cannot be inferred from the shapes of the respective transition fronts.

The evolution of (short duration) root mean square (RMS) chordwise velocity contours across the cross-section of the stationary crossflow vortex is shown in Figs. 8 through 10. The initial evolution in Fig. 8 is qualitatively analogous to that presented by Li et al. ${ }^{8}$ on the basis of their NPSE computations. When transition is completed (see image 4 in Fig. 9), the peak fluctuations have shifted to the nearwall region. However, a visible signature of the stationary vortex is still observed within the outer part of the boundary layer. The spanwise variations appear to become more diffuse as the turbulent flow evolves further downstream; yet they can still be observed even at $x / c=$ 0.578 (Fig. 10), i.e., more than 10 boundary-layer thickneses downstream of the peak mean wall shear.

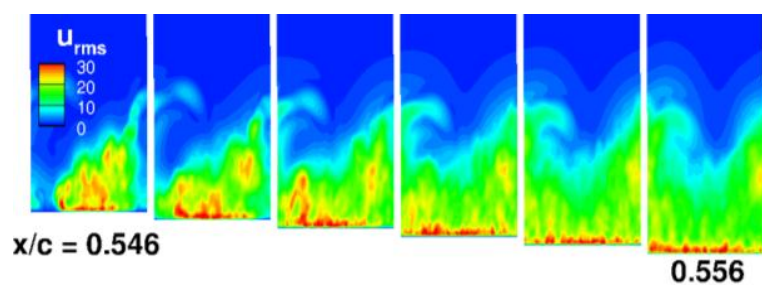

Fig. 9. RMS fluctuations in chordwise velocity component across the cross-section of the stationary vortex at selected stations $(0.544<x / c \leq$ 0.556).

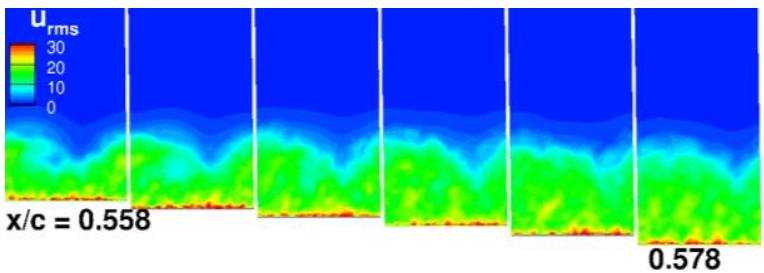

Fig. 10. RMS fluctuations in chordwise velocity component across the cross-section of the stationary vortex at selected stations $(0.556<x / c \leq 0.578)$.

Figure 11 shows an instantaneous snapshot of the flowfield at a selected instant of time. An isosurface of $u=$ $175 \mathrm{~m} / \mathrm{s}$ (i.e., approximately 71 percent of the edge velocity) is shown along with crossplane visualizations of temperature contours at three selected chordwise stations. Upstream of the transition onset, the isosurface indicates a series of ridges that are inclined with respect to the vortex axis and are centered along a spanwise location that approximately corresponds to the peak location of the secondary instability mode shape. The latter location lies approximately in-between the adjacent pair of locations where the boundary layer thickness has increased significantly as a result of the upwelling associated with a finite-amplitude crossflow vortex. As nonlinear effects come into play, the ridges develop a localised spanwise oscillation near the peak of the secondary instability mode shape (see the middle cut at $x / c=0.541$ in Fig. 11). Further downstream, this local deformation begins to spread out, particularly towards the wall and at approximately the same spanwise location relative to the axis of the stationary crossflow vortex. Since the peak of the Z-mode of secondary instability was below the crests of the overturning contours, the tertiary fluctuations can quickly begin to influence the nearwall dynamics, which then leads to the onset of self-sustaining turbulence. 


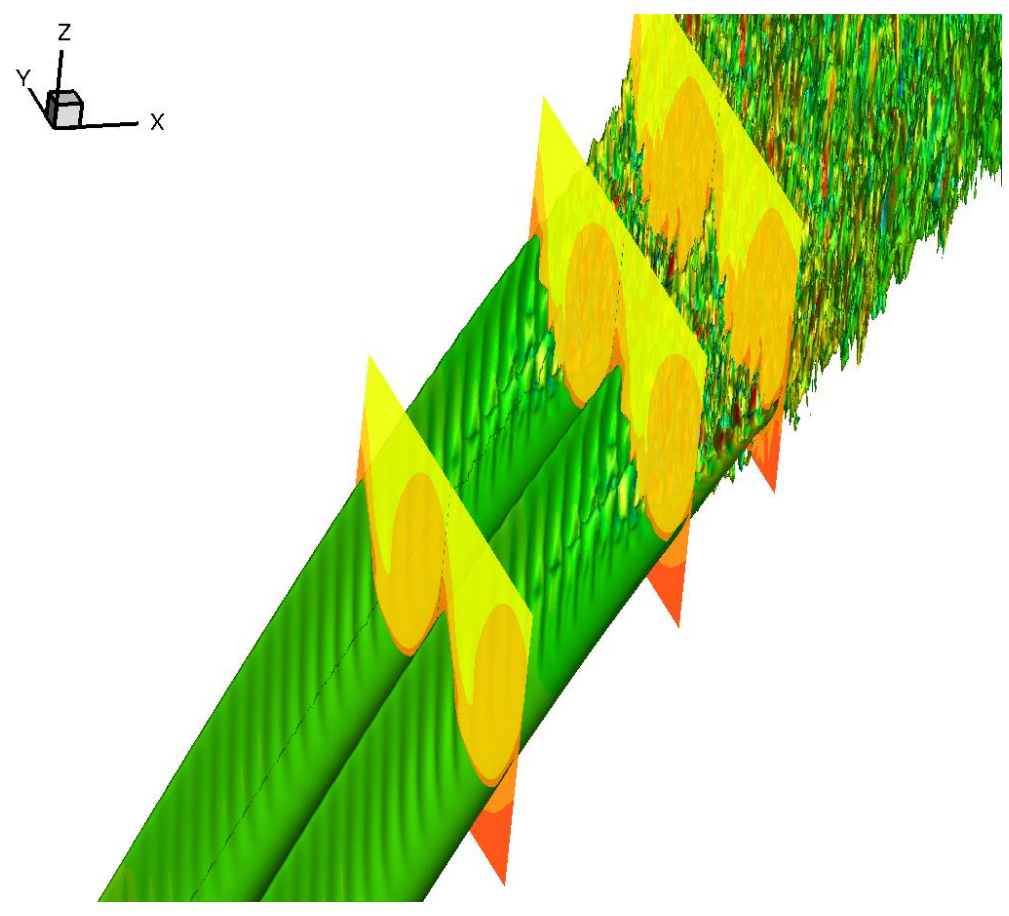

Fig. 11. Instantaneous isosurface of $u=175 \mathrm{~m} / \mathrm{s}$ along with temperature contours at selected $x$ locations $(x / c=0.527,0.541$, and 0.549 , respectively). The chordwise velocity for the isosurface is selected to coincide with the approximate location of peak chordwise velocity fluctuations associated with the secondary instability mode of interest.

\section{Secondary Instability of Traveling Crossflow Vortices}

In general, a swept-wing boundary layer can be susceptible to various types of primary instabilities such as attachment-line instability, ${ }^{49}$ stationary and traveling crossflow modes, and Tollmien-Schlichting (TS) waves. Regardless of which mode dominates the primary amplification stage, the other modes could play a role during the nonlinear stage and, hence, influence the onset of transition. Although it appears that the more likely scenario in cases of practical interest would involve the breakdown of stationary crossflow vortices, either via some type of high frequency secondary instability ${ }^{20,50-53}$ or through interactions involving TS waves or the traveling mode of crossflow instability, other scenarios involving a more significant role for the traveling crossflow instability ${ }^{54,55}$ may become relevant under less common circumstances. These may happen when either the initial amplitudes of traveling modes become sufficiently large as a result of higher amplitude freestream disturbances and/or when the surface has been carefully polished to minimize the initial amplitudes of stationary crossflow modes.

In a practical setting, it is perhaps more important to investigate the nonlinear breakdown scenarios associated with a mixed evolution of stationary and traveling crossflow modes. However, as a prelude to the more complex scenario, the secondary instability of traveling crossflow modes alone is studied herein to obtain some useful insights regarding their breakdown.

\section{A. Nonlinear Evolution of a Traveling Crossflow Mode}

Figure 12 shows $\mathrm{N}$-factor curves of crossflow instability of $8 \mathrm{~mm}$ spanwise wavelength for selected frequencies ranging from 0 to $2,250 \mathrm{~Hz}$. As expected, the most amplified traveling mode achieves higher $\mathrm{N}$-factors than the stationary mode; and, more importantly, the traveling mode with $f=1,500 \mathrm{~Hz}$ reaches $\mathrm{N}=10$ within nearly half the distance it takes for the stationary crossflow modes to reach the same $\mathrm{N}$-factor value. This indicates the utility of studying the breakdown of traveling waves to obtain a more complete picture of crossflow-dominated transition, especially in noisy environments. Since an $\mathrm{N}$-factor value of 10 is first reached by a traveling crossflow mode of $f=$ $1,500 \mathrm{~Hz}$, the latter mode is selected for the purpose of secondary instability analysis. 


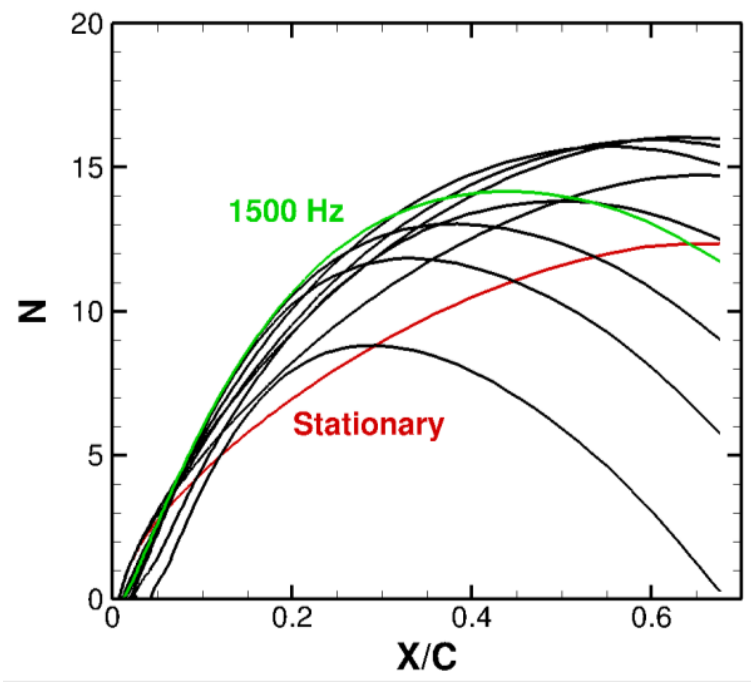

Figure 12. Linear $\mathrm{N}$-factors for crossflow instability modes with $\lambda=8 \mathrm{~mm}$ and frequency ranging from 0 to $2,250 \mathrm{~Hz}$. The $\mathrm{N}$-factor first reaches a value of 10 for the $1,500 \mathrm{~Hz}$ mode indicated by green curve. $\mathrm{N}$-factor curve for the stationary crossflow mode is shown in red.

Figure 13(a) compares the nonlinear development of stationary and traveling crossflow vortices of the same spanwise wavelength $(\lambda=8 \mathrm{~mm})$ and the same initial amplitude $\left(A_{\text {init }}=1 \times 10^{-5}\right)$. In the initial region of linear growth, the amplitudes of modes associated with the traveling crossflow vortex (green lines in Figure 13(a)), rise much faster than the stationary mode amplitudes (red lines in Figure 13(a)). As the amplitudes become larger, the traveling mode begins to saturate much earlier than the stationary mode, possibly due to the rapid rise in its mean flow correction, and ceases its growth beyond approximately $x / c=0.2$. On the other hand, the stationary mode does not saturate until approximately $x / c=0.5$. The traveling mode reaches the peak amplitude of approximately 0.16 before beginning to decay in an oscillatory fashion. On the other hand, the stationary mode grows monotonically over most of the streamwise domain and reaches a maximum amplitude of 0.32 , i.e., twice the peak amplitude achieved by the traveling mode. The above comparison between nonlinear evolution of isolated traveling and stationary crossflow disturbances is very similar to that reported by Malik et al ${ }^{14}$ for a different airfoil configuration. The maximum mean flow correction amplitudes for both stationary and traveling mode are relatively similar at 0.08 and 0.07 , respectively.

Modal amplitudes of traveling crossflow vortices of $1500 \mathrm{~Hz}$ are shown in Figure 13(b) for initial amplitudes of $2.5 \times 10^{-6}, 5.0 \times 10^{-6}, 7.5 \times 10^{-6}$ and $1 \times 10^{-5}$. Amplitudes of the mean flow correction and the first harmonic are also shown for the case of the smallest initial amplitude. The general features of oscillatory behavior and decay after saturation as seen earlier in Figure 13(a) for $A_{\text {init }}=1 \times 10^{-5}$ are present for all initial amplitudes, with waves corresponding to higher initial amplitudes exhibiting an earlier quasi-saturation. Figure 13(b) also indicates that traveling crossflow modes with higher initial amplitudes saturate at progressively smaller peak amplitudes.

When the initial amplitudes of the traveling and stationary crossflow amplitudes are equal, the stronger growth of the traveling mode leads to an earlier onset of secondary instability. Figure 14 presents a comparison of the secondary $\mathrm{N}$-factors for the stationary and traveling primary modes considered in Figure 13(a). Specifically, the Nfactor curves of $\mathrm{Y}$ - and Z-modes of secondary instability that first reach an $\mathrm{N}$-factor of 10 are plotted for both types of primary waves. The peak $\mathrm{N}$-factor for the Z-mode instabilities of the stationary crossflow vortex is less than 10; and, hence, the secondary Z-mode disturbance with $f=42.5 \mathrm{kHz}$ corresponding to the peak of the $\mathrm{N}$-factor envelope is shown in that case. In all cases the neutral points of the secondary instability appear to be in the neighborhood of the locations where the primary crossflow vortices begin to saturate (see Figure 13). The most dangerous secondary instability mode of the traveling crossflow vortex (i.e., a Y-mode) becomes unstable at a primary vortex amplitude of approximately 0.16 . Similarly, the corresponding mode in the stationary case (also a Y-mode) becomes unstable at nearly the same primary amplitude of approximately 0.17 . Over the same distance of approximately $20 \%$ chord length, the most unstable $\mathrm{Y}$-modes reach an $\mathrm{N}$-factor of over 30 in both the stationary and the traveling cases. However, the initial slope of the $\mathrm{Y}$-mode $\mathrm{N}$-factor curve for the traveling vortex is larger than that in the stationary case, therefore, the secondary instability reaches an $\mathrm{N}$-factor of 10 faster than than in the stationary case.

In the future, it will be useful to extend the secondary instability computations of traveling crossflow modes to even lower initial amplitudes, so as to provide insights regarding the threshold initial amplitude of traveling 
crossflow modes that could dominate the transition process.

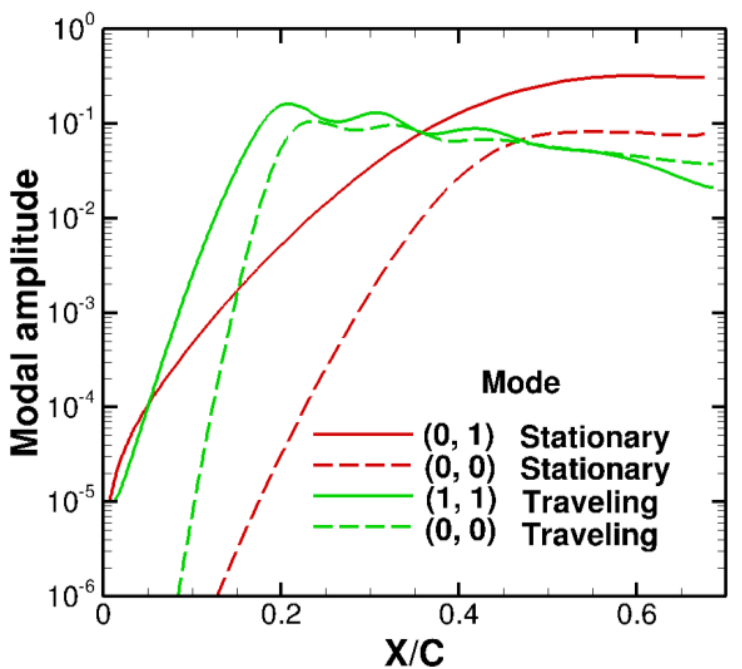

Fig. 13(a). Nonlinear evolution of stationary and traveling crossflow $(f=1500 \mathrm{~Hz}$ ) modes with $\lambda=8$ $\mathrm{mm}$ and the same initial amplitude $\left(A_{\text {init }}=1 \times 10^{-5}\right)$ : Comparison of modal amplitudes for the fundamental and mean flow correction modes.

B. Secondary Instability of traveling crossflow vortex

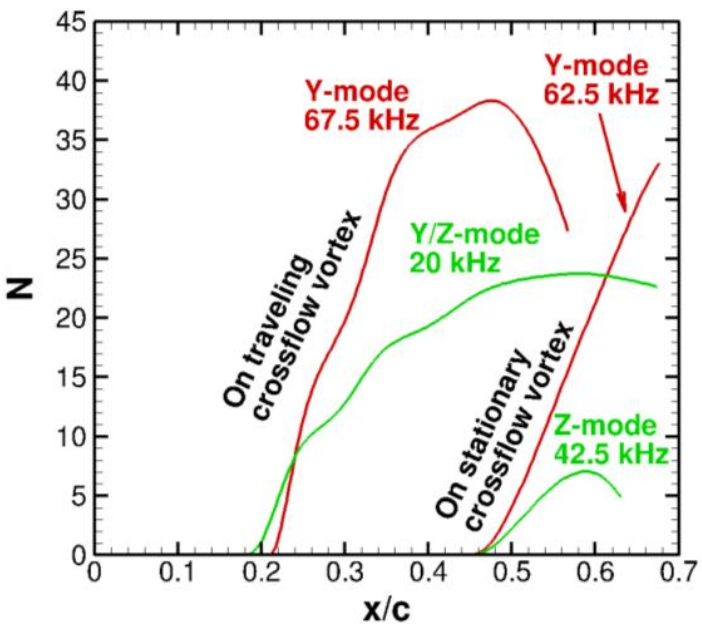

Figure 14. Comparison of $\mathrm{N}$-factor curves for most dangerous secondary instability modes of stationary and traveling crossflow vortices. The metric for growth potential corresponds to earliest attainment of $\mathrm{N}=10$ or the highest $\mathrm{N}$-factor when $\mathbf{N}=\mathbf{1 0}$ is never achieved. Both primary crossflow vortices have the same spanwise wavelength of 8 mm, and the traveling crossflow vortex has a frequency of $1500 \mathrm{~Hz}$.

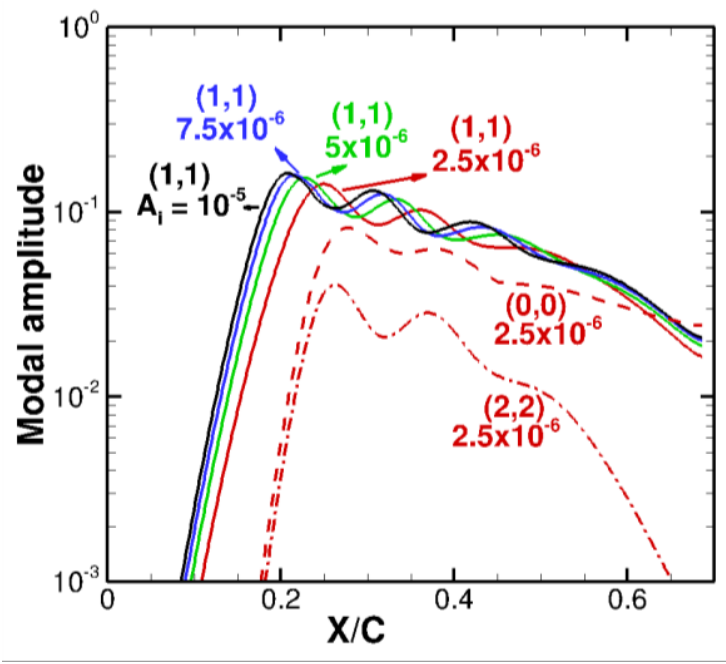

Fig. 13(b). Evolution of fundamental amplitude for traveling crossflow vortices with different initial amplitudes. The amplitudes of mean flow correction and the first harmonic are also shown for the case with the smallest initial amplitude $\left(A_{\text {init }}=2.5 \times 10^{-6}\right)$.

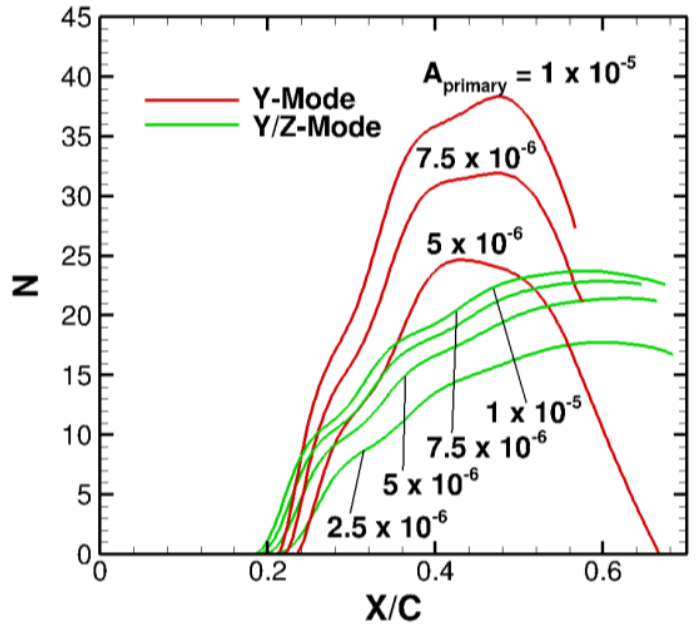

Fig. 15. Peak N-factors of secondary instability of traveling crossflow vortices of different initial amplitudes.

Figure 15 shows the $\mathrm{N}$-factor curves for secondary instability modes that first achieve an $\mathrm{N}$-factor of 10 for each initial amplitude of the traveling crossflow vortex, namely $A_{\text {init }}=2.5 \times 10^{-6}, 5.0 \times 10^{-6}, 7.5 \times 10^{-6}$, and $1 \times 10^{-5}$, respectively. Regardless of the initial primary amplitude in this case, the dominant secondary instability mode is a 


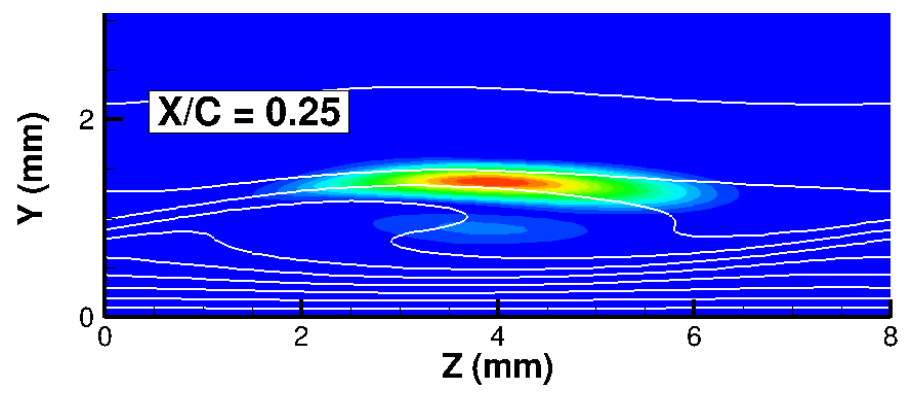

Fig. 16(a). Chordwise velocity eigenfunction for a Y-mode secondary instability of a primary traveling crossflow wave of initial amplitude $1 \times 10^{-5}$. Secondary instability frequency is $67.5 \mathrm{kHz}$.

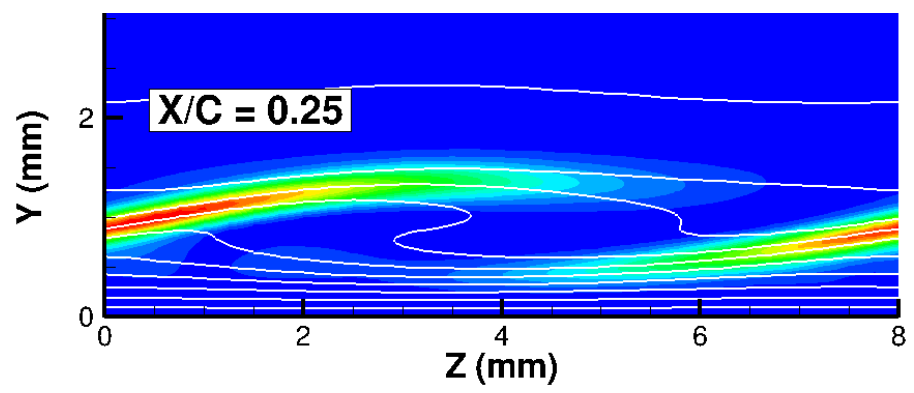

Fig. 16(b). Chordwise velocity eigenfunction for a $Y / Z$ mode secondary instability of a primary traveling crossflow wave of initial amplitude $1 \times 10^{-5}$. The frequency of the secondary instability mode is $20 \mathrm{kHz}$.

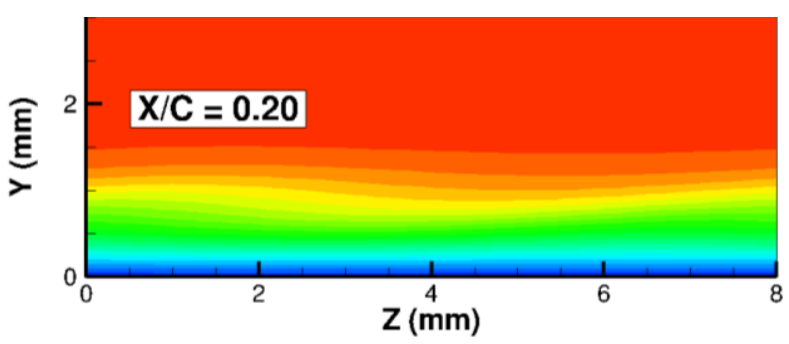

(a) $A_{\text {init }}=\mathbf{2 . 5} \times \mathbf{1 0}^{-6}$

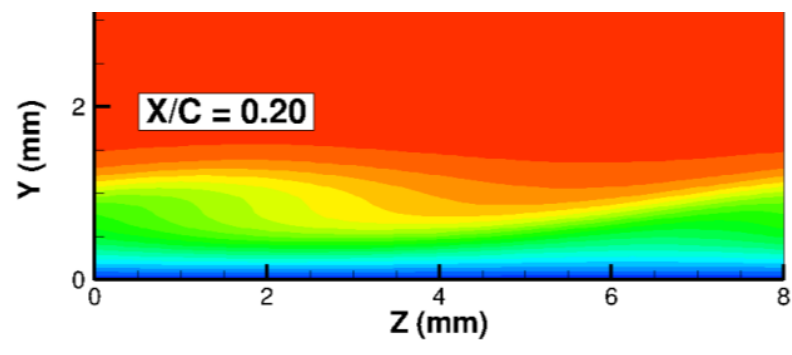

(c) $A_{\text {init }}=7.5 \times 10^{-6}$

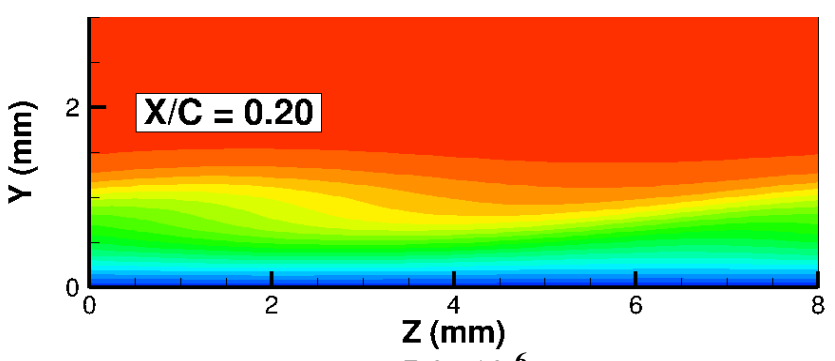

(b) $A_{\text {init }}=\mathbf{5 . 0 \times 1 0 ^ { - 6 }}$

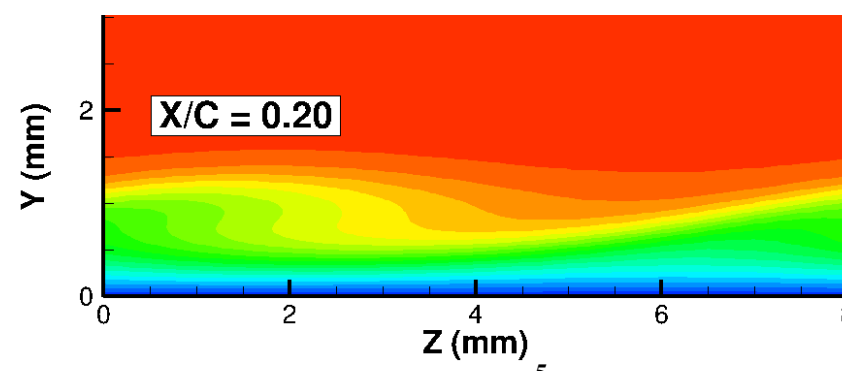

(d) $A_{\text {init }}=1.0 \times 10^{-5}$

Fig. 17. Chordwise velocity contours associated with a finite amplitude traveling crossflow mode. The coordinate system is assumed to translate with the traveling mode and the chordwise location of $x / c=0.20$ is chosen to be in the vicinity of the onset of secondary instabilities.

Y-Mode except for the case of lowest initial primary amplitude for which no Y-mode was found by the eigenvalue solver. The subdominant modes are of the mixed type, i.e., Y/Z modes that receive comparable contributions from 
both wall-normal and spanwise shear of the basic state. For the cases with larger values of initial primary amplitude, the peak $\mathrm{N}$-factors values for secondary instability are correspondingly larger as expected. While the $\mathrm{Y}$-modes decay rapidly after reaching their peak $\mathrm{N}$-factors, the Z-modes continue to grow almost monotonically. The frequency of $\mathrm{Y}$-mode disturbances achiving the highest $\mathrm{N}$-factor values ranges from $58-67.5 \mathrm{kHz}$, whereas the frequency range for the most amplified $\mathrm{Y} / \mathrm{Z}$ modes corresponds to $15-19 \mathrm{kHz}$.

Figs. 16(a) and 16(b) illustrate the chordwise velocity eigenfunctions of the Y mode and Y/Z mode, respectively, at $x / c=0.25$. In a coordinate frame that travels with the primary instability (i.e, the traveling crossflow mode), the basic state becomes steady in time. Figure 17(a) through 17(d) show the mean chordwise velocity contours in the traveling frame at $x / c=0.2$ for initial primary amplitudes of $2.5 \times 10^{-6}, 5.0 \times 10^{-6,} 7.5 \times 10^{-6}$ and $10^{-5}$, respectively. For the highest initial amplitude, the base velocity contours develop an overturning behavior similar to that seen in previous studies of finite amplitude stationary crossflow vortices. Given that the onset of secondary instability even for the lowest initial amplitude $\left(2.5 \times 10^{-6}\right)$ occurs near $x / c=0.2$, it may be inferred that even a relatively modest deformation of the underlying basic state can lead to the amplification of secondary modes.

\section{Concluding Remarks}

In this paper, previously reported holistic predictions of crossflow induced transition over a realistic swept airfoil configuration were extended via high fidelity computations involving the receptivity, secondary instability, and nonlinear breakdown stages of transition. The results presented herein demonstrate that the adjoint PSE capability of the LASTRAC code can be used to predict the excitation of stationary crossflow modes due to small amplitude surface roughness for a realistic airfoil configuration of relevance to future subsonic transports. Furthermore, the compressed length of the stable (i.e., subcritical) region upstream of the lower branch neutral location near the airfoil nose is shown to reduce the peak receptivity associated with nonlocalized, wavy-wall distributions of surface height variation. In future, the capability for receptivity prediction will be further extended to allow more rational predictions of the overall transition process for a given specification of the environmental disturbances.

Despite the substantial differences in mode shapes of the secondary instability modes associated with spanwise and wall-normal shear, the overall features of the transition pattern obtained herein for the spanwise mode are very similar to those obtained in a separate work focused on transition due to secondary instability modes driven by the wall-normal shear. Specifically, the transition front visualized via time averaged wall shear indicates a spanwise varying pattern resembling the sawtooth pattern visualized in previous wind tunnel experiments using naphthalene sublimation. Furthermore, in both cases, the memory of the initial stationary crossflow vortex persists even after transition. In the present computational domain, distinct spanwise inhomogeneity is observed even at distances of more than 20 boundary layer thicknesses past the location of peak skin friction. In other words, the ensembleaveraged turbulent flow varies along the spanwise direction throughout the computational domain. Since a drag prediction method based on the assumption of spanwise homogeneity of the first and second moment statistics will not capture these 3D effects, skin friction predictions based on Reynolds Averaged Navier-Stokes (RANS) equations may entail a nonzero bias in its predictions. Quantification of these effects associated with the relaxation of the spanwise perturbed turbulence will be a topic for future research.

The traveling crossflow vortices support secondary instabilities in much the same way as the stationary crossflow modes. Even though the traveling crossflow vortices saturate at much lower amplitudes than their stationary counterparts, the growth of secondary instabilities of the traveling crossflow vortices is at least as strong as the secondary modes of the stationary crossflow modes. The important issue of threshold initial amplitudes of the traveling vortices required to sustain a significant amplification of secondary instabilies will also be examined in future work.

The multiplicity of secondary instability modes with a significant growth potential implies that transition prediction based on secondary $\mathrm{N}$-factors must address the issues of mode selection from the competing instability mechanisms as well as the nonlinear interactions between multiple types of primary and secondary instabilities and the breakdown scenarios. An additional key question in the context of transition prediction for laminar flow technology corresponds to whether or not increasingly rational transition prediction can be achieved with easily available input data regarding the disturbance environment such as the surface finish and a small number of parameters characterizing the freestream disturbances.

\section{Acknowledgments}

This work was performed as part of the Revolutionary Computational Aerosciences discipline under the Aeronautical Sciences project of NASA's Fundamental Aeronautics Program. The authors would like to thank Prof. Pino Martin of the University of Maryland for providing the original version of the DNS code used in this effort. 


\section{References}

${ }^{1}$ Collier, F., "Subsonic Fixed Wing Project Overview," Proceedings of NASA FAP 2008 Annual Meeting, Atlanta, Oct. 7-9, 2008.

${ }^{2}$ Report of the Group of Personalities, "European Aeronautics: A Vision for 2020," Office for Official Publications of the European Communities, January 2001.

${ }^{3}$ Collier, F., "NASA Sponsored Activities in Laminar Flow Technologies for Advanced Transport Aircraft," 1st UTIASMITACS International Workshop on Aviation and Climate Change, Toronto, May 27-28, 2010.

${ }^{4}$ Saric, W. S., Carillo, R. B., and Reibert, M. S., "Leading Edge Roughness as a Transition Control Mechanism," AIAA Paper 98-0781, 1998

${ }^{5}$ Carpenter, A. L., Saric, W. S., and Reed, H. L., "Laminar Flow Control on a Swept Wing with Distributed Roughness," AIAA Paper 2008-7335, 2008.

${ }^{6}$ Rhodes, R. G., Carpenter, A. L., Reed, H. L., and Saric, W., "CFD Analysis of Flight-Test Configuration for LFC on Swept Wings," AIAA Paper 2008-7336, 2008.

7 Belisle, M., Neale, T., Reed, H., and Saric, W. "Design of a Swept-Wing Laminar Flow Control Flight Experiment for Transonic Aircraft," AIAA paper 2010-4381, 2010.

${ }^{8}$ Li, F., Choudhari, M. M., Carpenter, M. H., Malik, M. R., Chang, C.-L. and Streett, C. L., "Roughness Based Crossflow Transition Control for a Swept Airfoil Design Relevant to Subsonic Transports," AIAA Paper 2010-4380, 2010.

${ }^{9}$ Belisle, M. J., Roberts, M. W., Tufts, M. W., Tucker, A. A., Williams, T., Saric, W. S., and Reed, H. L., "Design of the Subsonic Aircraft Roughness Glove Experiment (SARGE),” AIAA Paper 2011-3524, 2011.

${ }^{10}$ Malik, M., Liao, W., Lee-Rausch, E., Li, F., Choudhari, M., and Chang, C.-L., "Computational Analysis of the G-III Laminar Flow Glove," AIAA Paper 2011-3525, 2011.

${ }^{11}$ Hartshorn, F., Belisle, M. J., and Reed, H. L., "Computational Optimization of a Natural Laminar Flow Experimental Wing Glove," AIAA Paper 2012-0870, 2012.

${ }^{12}$ Liao, W., Malik, M. R., Lee-Rausch, E. M., Li, F., Nielsen, E. J., Buning, P. G., Chang, C. L., and Choudhari, M., "Boundary-Layer Stability Analysis of the Mean Flows Obtained Using Unstructured Grids," AIAA Paper 2012-2690, 2012.

${ }^{13}$ Roberts, M. W., Reed, H. L., and Saric, W. S., "A Transonic Laminar-Flow Wing Glove Flight Experiment: Computational Evaluation and Linear Stability," AIAA Paper 2012-2668, 2012.

${ }^{14}$ Malik, M. R., Liao, W., Li, F., and Choudhari, M., "DRE-Enhanced Swept-Wing Natural Laminar Flow at High Reynolds Numbers," AIAA Paper 2013-412, 2013.

${ }^{15}$ Saric, W. S., "Flight Experiments on Swept-Wing Roughness Receptivity: Phase 2. Feasibility of a Laminar Flow Flight Test," Phase 2, Year 1 Draft Report for AFOSR Grant FA955008-1-0475, June, 2009.

${ }^{16}$ Campbell, R. L., Pandya, M. J., and Campbell, M. L., "An Efficient Approach to Natural Laminar Flow Design," Presentation at the Royal Aeronautical Society Aerodynamics Conference, July 2010, CD.

${ }^{17}$ Crouch, J. D., Sutanto, M., I., Witkowski, D. P, Watkins, A. N., Rivers, M. B, and Campbell, Richard L, "Assessment of the National Transonic Facility For Laminar Flow Testing," AIAA 2010-1302, 2010.

${ }^{18}$ The Aeronautical Sciences Project, http://www.aeronautics.nasa.gov/fap/aeronautical_sciences.html, Checked on April 27, 2013.

${ }^{19}$ Choudhari, M., Chang, C.-L., Streett, C. L., and Balakumar, P., "Integrated Transition Prediction: A Case Study in Supersonic Laminar Flow Control,” AIAA Paper 2003-0973, 2003.

${ }^{20}$ Malik, M. R., Li, F., Choudhari, M., and Chang, C.-L., "Secondary Instability of Crossflow Vortices and Swept-Wing

Boundary-Layer Transition,” J. Fluid Mech., Vol. 399, 1999, pp. 85-115.

${ }^{21}$ Janke, E., "Receptivity and Transition Control of Swept-Wing Boundary-Layers: Effects of Surface Curvature and Nonlinearity," AIAA Paper 2001-2980, 2001.

${ }^{22}$ Chang, C.-L. and Choudhari, M. M., "Boundary-Layer Receptivity and Integrated Transition Prediction," AIAA Paper 2005-0526, 2005.

${ }^{23}$ Choudhari, M, Chang, C.-L. and Jiang, L., "Towards Transition Modeling for Supersonic Laminar Flow Control," Philosophical Transactions of Royal Society of London (Physical and Mathematical Sciences), Vol. 363, No. 1830, 2005, pp. 1041-1259.

${ }^{24}$ Li, F. and Choudhari, M., "Spatially Developing Secondary Instabilities and Attachment Line Instability in Supersonic Boundary Layers," AIAA Paper 2008-590, 2008.

${ }^{25}$ Li, F., Choudhari, M., Chang, C.-L., Streett, C. L., and Carpenter, M. H., "Roughness Based Crossflow Transition Control: A Computational Assessment," AIAA Paper 2009-4105, 2009.

${ }^{26} \mathrm{Li}$, F., Choudhari, M. M., Chang, C.-L. Streett, C. L., and Carpenter, M. H., "Computational Modeling of Roughness-Based Laminar Flow Control on a Subsonic Swept Wing,” AIAA J., Vol. 49, No. 3, March 2011, pp. 520-529.

${ }^{27}$ Duan, L., Choudhari, M., and Li, F., "Direct Numerical Simulation of Crossflow-Induced Transition in a Swept Wing Boundary Layer," To be presented at 21 ${ }^{\text {st }}$ AIAA Computational Fluid Dynamics Conference, San Diego, CA, June 23-27, 2013.

${ }^{28}$ Wie, Y.-S., "BLSTA - A Boundary Layer Code for Stability Analysis," NASA CR 4481, 1992.

${ }^{29}$ Chang, C.-L., "Langley Stability and Transition Analysis Code (LASTRAC) Version 1.2 User Manual," NASA/TM-2004213233, June, 2004.

${ }^{30}$ Chang, C.-L. and Choudhari, M., "Boundary layer Receptivity and Integrated Transition Prediction,” AIAA Paper 2005-526, 2005. 
${ }^{31}$ Jiang, L., Choudhari M., Chang, C., and Liu, C., "Direct Numerical Simulations of Crossflow Disturbances in Supersonic Boundary Layers," AIAA Paper 2004-589, 2004.

${ }^{32}$ Jiang, G. S. and Shu, C. W., "Efficient Implementation of Weighted ENO Schemes," Journal of Computational Physics, Vol. 126, No. 1, 1996, pp. 202-228.

${ }^{33}$ Taylor, E. M., Wu, M., and Martin, M. P., "Optimization of Nonlinear Error Sources for Weighted Non-Oscillatory Methods in Direct Numerical Simulations of Compressible Turbulence," Journal of Computational Physics, Vol. 223, No. 1, 2006, pp. 384-397.

${ }^{34}$ Martin, M. P., Taylor, E. M., Wu, M., and Weirs, V. G., “A Bandwidth-Optimized WENO Scheme for the Effective Direct Numerical Simulation of Compressible Turbulence," J. Comp. Phys., Vol. 220, 2006, pp. 270-289.

${ }^{35}$ Williamson, J., "Low-Storage Runge-Kutta Schemes," Journal of Computational Physics, Vol. 35, No. 1, 1980, pp. 48.

${ }^{36}$ Thompson, K. W., "Time Dependent Boundary Conditions for Hyperbolic Systems,” Journal of Computational Physics, Vol. 68, No. 1, 1987, pp. 1-24.

${ }^{37}$ Duan, L., Beekman, I., and Mart'1n, M. P., "Direct Numerical Simulation of Hypersonic Turbulent Boundary Layers. Part 3: Effect of Mach Number," Journal of Fluid Mechanics, Vol. 672, 2011, pp. 245-267.

${ }^{38}$ Choudhari, M. and Streett, C. L., “Theoretical Prediction of Boundary-Layer Receptivity,” AIAA Paper 1994-2223, 1994.

${ }^{39}$ Crouch, J. D., "Receptivity of Boundary Layers," AIAA Paper 1994-2224, 1994.

${ }^{40}$ Goldstein, M. E., "Scattering of Acoustic Waves into Tollmien-Schlichting Waves by Small Streamwise Variations in Surface Geometry," J. Fluid Mech., Vol. 154, pp. 509-529, 1985.

${ }^{41}$ Ruban, A. I., "On the Generation of Tollmien-Schilichting Waves by Sound," Fluid Dyn., Vol. 19, pp.709-716, 1985.

${ }^{42}$ Choudhari, M. and Streett, C. L., "Boundary Layer Receptivity Phenomena in Three-Dimensional and High-Speed Boundary Layers," AIAA Paper 1990-5258, 1990.

${ }^{43}$ Choudhari, M., "Roughness-Induced Generation of Crossflow Vortices in Three-Dimensional Boundary Layers," Theor. and Comp. Fluid Dyn., Vol. 5, 1994, pp. 1-31.

${ }^{44}$ Carpenter, M. H., Choudhari, M. M., Li, F., Streett, C. L. and Chang, C.-L., "Excitation of Crossflow Instabilities in a Swept Wing Boundary Layer," AIAA Paper 2010-378, 2010.

${ }^{45}$ Collis, S. S. and Lele, S. K., "Receptivity to Surface Roughness Near a Swept Leading Edge," J. Fluid Mech., Vol. 380, 1999, pp. 141-168.

${ }^{46}$ White, E., and Saric, W. S., "Secondary Instability of Crossflow Vortices,” J. Fluid Mech., Vol. 525, 2005, pp. $275-308$.

${ }^{47}$ Bonfigli, G, and Kloker, M. "Secondary Instability of Crossflow Vortices: Validation of the Stability Theory by Direct Numerical Simulation," J. Fluid Mech., Vol. 583, 2007, pp. 229-272.

${ }^{48}$ Huai, X., Joslin, R. D., and Piomelli, U., "Large-Eddy Simulation of Transition to Turbulence in a Swept Wing Boundary Layer," AIAA Paper 1997-0750, 1997.

${ }^{49}$ Poll, D.I.A., "Transition in the Infinite Swept Attachment Line Boundary Layer,” The Aeron. Quarterly, Vol XXX, 1979, pp. 607-629.

${ }^{50}$ Kohama, Y., Saric, W. S., and Hoos, J. A., “A High-Frequency Secondary Instability of Crossflow Vortices that Leads to Transition," in Boundary Layer Transition and Control, Proc. Royal Aeronautical Society, Cambridge, 8-12 April, 1991, London, UK.

${ }^{51}$ Balachandar, S., Streett, C. L., and Malik, M. R., "Secondary Instability in a Rotating Disk Flow," J. Fluid Mech., Vol. 242, 1992, pp. 323-347.

${ }^{52}$ Malik, M. R., Li, F., and Chang, C.-L., "Crossflow Disturbances in Three-Dimensional Boundary Layers: Nonlinear Development, Wave Interaction and Secondary Instability," J. Fluid Mech., Vol. 268, 1994, pp. 1-36.

${ }^{53}$ Wassermann, P. and Kloker, M., "Mechanisms and Passive Control of Crossflow-Vortex-Induced Transition in a ThreeDimensional Boundary Layer," J. Fluid Mech., Vol. 456, 2002, pp. 49-84.

${ }^{54}$ Bippes, H., "Basic Experiments on Transition in Three-Dimensional Boundary Layers Dominated by Crossflow Instability," Progress in Aerospace Sciences, Vol. 35, 1999, pp. 363-412.

${ }^{55}$ Wassermann, P. and Kloker, M., "Transition Mechanisms Induced by Traveling Crossflow Vortices in a Three-Dimensional Boundary Layer,” J. Fluid Mech., Vol. 483, 2003, pp. 67-89. 